\title{
Tipos de humor en la publicidad impresa en Colombia
}

\section{Carolina María Cifuentes* \\ Universidad de Los Andes}

Recibido: enero 11 de 2005

Revisado: enero 17 de 2005

Aceptado: febrero 1 de 2005

\section{Resumen}

Una de las herramientas frecuentemente utilizadas por los publicistas para persuadir es el humor. De ahí el creciente interés por estudiar su influencia en el procesamiento, actitudes y comportamiento de los individuos. Se realizó una revisión de investigaciones sobre humor publicitario y sus efectos en la persuasión, atención, comprensión, actitudes y memoria, así como estudios sobre humor y factores culturales, de audiencia, medios masivos y tipos de producto. Se presenta una tipología de publicidad humorística impresa colombiana, producto de revisiones bibliográficas y del seguimiento del humor publicitario a través del tiempo en la revista Semana, identificando tres nuevas categorías. Los tipos de humor propuestos son: incongruencia, hostil, sorpresa, juego de palabras, personificación, frases, alusiones, comparación, rimas, caricaturas, obras y exageración.

Palabras clave: publicidad, humor en publicidad, psicología, tipos de humor, actitudes.

\section{Abstract}

Humor is one of the most frequently used tools to persuade in advertising. Therefore, interest in studying

* Correspondencia: Carolina María Cifuentes. Correo electrónico: carolinamari@yahoo.com 
humor's influence in the attitudes and behavior of individuals has increased. A revision of research projects regarding humor in advertising and its effects in persuation, attention, comprehension, attitudes and memory was performed as well as studies concerning humor and cultural factors, type of audience, media and type of product. A classification of humorous advertisement published in Colombia has been executed on account of bibliographical revisions and of the serialized tracking of the Semana Magazine; leading to the identification of three new categories. The proposed types of humor are: incongruity, hostile, surprise, pun, personification, phrases, allusions, comparation, rhymes, cartoons, skits and exaggeration.

Index terms: advertising, humor in advertising, psychology, types of humor, attitudes.

\section{Introducción}

La publicidad contemporánea es cada vez más competitiva; busca captar la atención de los consumidores a como dé lugar, valiéndose de diversas técnicas que han demostrado ser apropiadas para este objetivo. El humor es una de esas herramientas frecuentemente implementadas por los creativos para persuadir, informar y divertir a todo tipo de consumidores.

Teniendo en cuenta la cantidad de información a la cual el hombre moderno es expuesto a diario en los medios de comunicación, es importante diseñar y desarrollar campañas publicitarias atractivas y fáciles de recordar para los consumidores. Con este fin, muchas agencias de publicidad hacen uso de frases, situaciones e imágenes graciosas como estrategia principal de sus campañas.

Aunque la técnica humorística ha estado presente en el ámbito publicitario por muchos años, desde la década de los 80 su utilización se ha incrementado significativamente en la publicidad norteamericana. Se estima que el $24 \%$ de los comerciales televisivos y el $31 \%$ de las cuñas radiales en Estados Unidos contienen alguna forma de humor (Weinberger y Campbell, 1991). La efectividad y la gran acogida del humor publicitario han llevado a que anualmente se inviertan billones de dólares en campañas que lo utilizan como técnica principal (Weinberger, Spotts, Campbell y Parsons, 1995).

Ante el incremento en el uso de la técnica humorística como herramienta publicitaria y las grandes cantidades de dinero que se invierten diariamente en este tipo de campañas, surge la necesidad de conocer a fondo la influencia del humor en las actitudes de los consumidores, así como las implicaciones de su uso en el ámbito de la publicidad.

A diferencia de otros aspectos ampliamente estudiados por la psicología del consumidor, las investigaciones realizadas acerca del efecto del humor en áreas como la persuasión, la capacidad para llamar la atención, la comprensión del mensaje y las actitudes hacia la marca se iniciaron hace tan solo tres décadas. Dichos estudios son relativamente pocos y se han realizado en contextos culturales diferentes al nuestro, como Estados Unidos y Europa. Este interés reciente implica que se han profundizado pocas variables que pueden ser afectadas por el humor, dejando todavía un amplio campo por explorar (Weinberger, Spotts, Campbell y Parsons, 1995). 
Una recopilación de estudios efectuados por varios autores ha corroborado que el humor tiene influencia en aspectos relacionados con la comunicación del mensaje y las actitudes del consumidor, la cual varía según los productos publicitados y los factores culturales. El presente artículo sintetiza los aspectos relevantes de estos estudios y aporta una tipología de humor en publicidad impresa colombiana, producto de una investigación realizada durante dos años acerca del tema.

\section{Investigaciones acerca del humor publicitario}

\section{Humor y persuasión}

El cambio de actitudes que tanto añoran los publicistas en estos tiempos es obtenido fácilmente cuando se implementan técnicas humorísticas en comerciales. Esto sucede debido a la capacidad persuasiva del humor, el cual genera un afecto positivo en el consumidor, que posteriormente es asociado con la marca publicitada (Lyttle, 2001). Así mismo, esta técnica bloquea el procesamiento central, haciendo que el consumidor de grandes argumentos se distraiga y sea más fácil persuadirlo (Madden y Weinberger 1982; Zhang, 1996; Lyttle, 2001).

Un estudio realizado por Chattopadhyay y Basu (1989) demostró que el humor en publicidad tiene un efecto moderadamente positivo en la persuasión del consumidor. Los sujetos con actitudes positivas previas hacia la marca fueron persuadidos en mayor medida por el humor publicitario que quienes tenían actitudes negativas previas. Igualmente, Scott, Klein y Briant (1990) investigaron el efecto del humor en la persuasión y encontraron que los sujetos expuestos a tratamientos humorísticos fueron persuadidos en mayor medida a acudir a un evento social en comparación con aquéllos expuestos a otros tipos de promociones.

\section{Humor y atención}

El primer objetivo que debe lograrse para que una publicidad sea efectiva es captar la atención del consumidor. Si un anuncio no llama la atención del individuo, éste hace caso omiso del mismo y se pierde todo el esfuerzo invertido en la comunicación realizada por los publicistas. En investigaciones desarrolladas en publicidad televisiva (Stewart y Furse, 1986), radial (Weinberger y Campbell, 1991) e impresa (Madden y Weinberger, 1982) se encontró que el humor tiene un efecto positivo en la atención al comercial.

Estudios realizados alrededor del tema confirman que el humor, como agente novedoso en el anuncio, atrae la atención de la audiencia en mayor medida que otros elementos presentes en anuncios no humorísticos (Sternthal y Craig, 1973, Madden y Weinberger, 1982; Madden y Weinberger, 1984; Madden y Weinberger, 1991). Adicionalmente, en investigaciones sobre el área educativa se ha demostrado el efecto positivo del humor sobre la atención de los individuos (Powell y Andersen, 1985; Bryant y Zillman, 1979).

Los efectos positivos del humor en la atención están mediados por la relación existente entre este último y el producto ofrecido. En investigaciones realizadas por Duncan (1979), Lull (1940) y Madden y Weinberger (1982) se demostró que el humor directamente relacionado con el producto publicitado tiene un mayor efecto en la atención, en comparación con el humor que no se relaciona con el objeto promocionado. Por su parte, Weinberger y Gulas (1992) y Smith (1993) concuerdan con este planteamiento y añaden que el humor relacionado con el producto también puede producir un efecto de distracción en el procesamiento, el cual lleva a que el consumidor se enfoque en el humor del anuncio mas no en los argumentos que éste proporciona ni en la marca promocionada.

Madden y Weinberger (1982) encontraron que la relación positiva entre el humor y la atención depende de las características raciales y de géne- 
ro de la audiencia. Estos autores realizaron un estudio sobre los efectos del humor publicitario en la atención, en el que los hombres obtuvieron puntajes de atención más altos que las mujeres, al igual que las personas de raza blanca en comparación con las afroamericanas.

\section{Humor y comprensión}

Es indispensable que el consumidor comprenda el mensaje transmitido por la publicidad a la cual es expuesto; por esta razón, resulta necesario establecer los efectos de la publicidad humorística sobre la comprensión de los anuncios; los hallazgos se encuentran divididos.

Algunas investigaciones han demostrado que el uso del humor en comerciales facilita la comprensión del mensaje transmitido, en tanto que genera activación a procesar la información recibida y a comprenderla en menor tiempo (Weinberger y Gulas, 1992; Cantor y Venus, 1980; Duncan, Nelson y Frontczak, 1983; Zhang y Zinkhan, 1991). No obstante, otros autores han encontrado relaciones negativas entre el humor y la comprensión; relaciones que demuestran que éste confunde al consumidor e impide la total comprensión del mensaje presentado (Cantor y Venus 1980; Lammers, 1983; Sutherland y Middelton, 1983; Gelb y Zinkhan, 1986). Esta dualidad entre hallazgos puede explicarse por:

Falta de una definición consistente para la "comprensión".

Tipo de humor utilizado puede influir en la comprensión del anuncio.

Tipo de producto publicitado en el impacto del humor sobre la comprensión del mensaje.

\section{Humor y memoria}

Uno de los propósitos principales de la publicidad es hacer que las marcas anunciadas sean recordadas y reconocidas por los consumidores en el momento de la compra. De nada sirve desarrollar campañas graciosas y agradables si los consumidores no logran recordar qué producto o marca se estaba ofreciendo.

Según Nord y Meter (1980) (citado por Chung y Zhao, 2003, p. 123), los efectos del humor en el recuerdo y el reconocimiento se explican desde la teoría del condicionamiento, mediante la cual el humor es una recompensa por atender a un mensaje publicitario. Así, el humor actúa como reforzador positivo, causando una activación emocional que fomenta la memoria. Por consiguiente, un anuncio con contenido humorístico será más recordado y reconocido que uno publicitado sin humor.

Weinberger y Campbell (1991) demostraron que el humor presente en la publicidad tiene un efecto positivo en la memoria y en el reconocimiento de marca. De la misma forma, Chung y Zhao (2003) realizaron un estudio acerca de la relación entre comerciales humorísticos y memoria de marca. Encontraron que los sujetos recordaban más las marcas publicitadas con humor que las que estaban presentes en comerciales no humorísticos. Esta relación positiva fue mucho más fuerte cuando se trataba de productos de bajo involucramiento. Por su parte, Furnham (1998) investigó la relación entre el humor y el recuerdo, encontrando que la técnica humorística tiene un efecto positivo en el recuerdo de anuncios publicitarios.

Berg (2001) desarrolló un estudio que tenía como objetivo medir el reconocimiento de marca como una función del humor en la publicidad. De acuerdo con los análisis, el humor no afectó el grado de reconocimiento para marcas, aunque los participantes mostraron más asociaciones entre marcas y tipos de producto cuando fueron presentados al consumidor en comerciales humorísticos.

Por otro lado, Heckler y Childers (1992) propusieron que el poder recobrar la información de un anuncio difiere entre la información congruente y la incongruente, además postularon que la información no esperada produce una mejor recor- 
dación que la información esperada, y que la información relevante se recuerda más fácilmente que la irrelevante. Según este planteamiento, se podría hipotetizar que los comerciales que implementen un tipo de humor incongruente serán recordados en mayor medida que aquellos que contengan otro tipo de humor.

\section{Humor y actitudes}

El humor presente en la publicidad tiene efectos importantes en las evaluaciones que hacen los consumidores tanto de los anuncios como de las marcas ofrecidas en ellos. La influencia del humor en la actitud hacia el anuncio ha sido estudiada ampliamente; diversas investigaciones demuestran que se puede predecir la actitud hacia la marca a través de las evaluaciones del comercial que la publicita (Pikett, 1983; Mitchell y Olson, 1981; Zhang, 1996; Aitsech, Clines y Kellaris 2003).

En estudios realizados por Pikett (1983), Belch y Belch (1984), Chung y Zhao (2003), Aitsech, Clines y Kellaris (2003) se encontró una relación positiva entre el humor y la actitud hacia el anuncio. Con los resultados de estas investigaciones se demostró que el humor publicitario está estrechamente relacionado con el gusto por los comerciales. Se ha descubierto que la actitud hacia la marca, entendida como las evaluaciones positivas y negativas de los consumidores sobre ésta (Zhang, 1996), es más positiva cuando el anuncio que la promociona es humorístico. Esto indica que la actitud positiva hacia el anuncio generada por el humor es trasladada a la marca que se publicita (Gelb y Pikett, 1983; Ray y Batra (1983); Holbrook y O'Shaughnessy (1984); Lyttle, 2001).

Igualmente, Mitchel y Olson (1981), Lutz (1985), MacKenzie (1986) y Zhang (1996) demostraron que la actitud positiva hacia la marca se logra en mayor medida cuando existe una actitud positiva hacia el anuncio, lo cual es muy probable de conseguir si se implementan técnicas humorísticas en la ejecución de la publicidad.

\section{Humor y características del producto}

Los efectos del humor en la publicidad varían de acuerdo con la naturaleza del producto que se promociona. Campbell, Parsons, Spotts y Weinberger (1995), Duncan y Nelson (1985), Campbell y Weinberger (1991) clasificaron los productos presentes en el mercado de acuerdo con el involucramiento (alto/bajo) y con el tipo de procesamiento cognitivo (sentimiento/pensamiento). Las categorías se establecieron de la siguiente forma:

- Bajo involucramiento/sentimiento: cerveza, vino, alcohol, soda, chicles, dulces, comidas rápidas, tabaco, café, snacks, etc.

- Bajo involucramiento/pensamiento: productos de aseo del hogar, gasolina, drogas, tarjetas de crédito, medicamentos.

- Alto involucramiento/sentimiento: ropa, accesorios, perfumes, joyas, motos, tinturas para el pelo, cosméticos, equipos de audio, entre otros.

- Alto involucramiento/pensamiento: carros, seguros de vida, préstamos bancarios, máquinas, computadores, casas, llantas de carros, etc.

En un estudio realizado por Madden y Weinberger (1984) se encontró que el humor es más efectivo cuando se utiliza en anuncios que publiciten productos de bajo involucramiento/sentimiento, como dulces y bebidas. El humor publicitario es especialmente eficaz para este caso porque actúa como elemento diferenciador dentro de una categoría en la cual todos los productos son similares (Sylvester, 2002).

Weinberger y Campbell (1991) demostraron que el humor no es efectivo cuando se publicitan artículos de alto involucramiento/pensamiento, pues distrae al consumidor, quien para elegir este tipo 
de productos necesita procesar la información del anuncio y evaluar los argumentos presentes en el mismo. Además, hallaron que para productos de alto involucramiento que incluyen sentimiento, el humor relacionado con éstos es más eficaz que el humor no relacionado. Diversos estudios han demostrado que el humor es más efectivo en publicidad cuando se relaciona con el producto ofrecido (Kaplan y Pascoe, 1977; Madden, 1982; Weinberger y Campbell, 1991).

Por otra parte, Stewart y Furse (1986) encontraron que la publicidad humorística es más exitosa para productos existentes en el mercado que para nuevos productos.

\section{Humor y medios masivos}

En la actualidad, la publicidad humorística aparece diariamente en todos los medios de comunicación masiva. Por esta razón, durante las últimas décadas se han desarrollado investigaciones en torno al tipo de medio en el cual se presenta el humor publicitario (radio, televisión, prensa y magacines) y al contexto en el que aparece el anuncio.

Se ha demostrado que implementar humor en la publicidad es más efectivo para algunos medios de comunicación que para otros (Weinberger y cols, 1995). En Estados Unidos, por ejemplo, la publicidad humorística es más efectiva en radio y televisión que en medios impresos (Madden y Weinberger, 1984). Estos hallazgos son ratificados por Weinberger y Campbell (1991), quienes encontraron que el $30,6 \%$ de la publicidad norteamericana realizada en radio, el $24.4 \%$ en televisión y el $9.9 \%$ en medios impresos emplea el humor como técnica persuasiva exitosa en sus campañas. Igualmente, Weinberger y cols (1995) encontraron publicidad humorística en un porcentaje de $37.9 \%$ para televisión, $18.1 \%$ en medios impresos y $40.6 \%$ para radio.

Cuando el consumidor ve televisión, es considerado como un ente pasivo en un medio activo, mientras que en los periódicos y revistas es un ente activo interactuando con un medio pasivo. A partir de lo anterior se puede explicar el mayor uso de humor publicitario en televisión. Teniendo en cuenta que la información presentada al televidente no es escogida en su totalidad por éste, resulta más eficaz para los anunciantes publicitar en este medio, ya que el individuo es expuesto a un gran número de comerciales y por lo tanto hay más probabilidades de persuadirlo.

\section{Humor y factores culturales}

Es importante tener en cuenta las diferencias entre culturas en materia de humor para desarrollar estrategias apropiadas de acuerdo con el entorno cultural para el público al cual va dirigida la campaña; el humor es un fenómeno que ha estado presente en todas las culturas a lo largo de la historia. Al generar una reacción similar en individuos de regiones y culturas diferentes, es posible afirmar que el humor es un fenómeno universal (Weinberger, 1991). Sin embargo, estudios han encontrado que las preferencias sobre los tipos de humor y la forma de manejar la publicidad humorística difieren según la cultura (Whipple y Courtney, 1981 y Weinberger, 1991).

Aunque la percepción del humor se encuentra presente en todas las personas, se ha demostrado que los niveles de humor percibidos (bajo/moderado/ alto) son diferentes de acuerdo con la cultura a la cual pertenezcan (Weinberger y Campbell, 1991). Ahora bien, teniendo en cuenta que el lenguaje empleado en ciertas culturas difiere, se podría pensar que el humor es percibido de forma distinta en cada contexto.

\section{Humor percibido}

Es importante tener en cuenta que el humor es puramente subjetivo. Un mensaje que para muchas personas resulta humorístico, a otras no les causará ninguna gracia. Anteriormente, los investigadores no consideraban este aspecto y asumían implícitamente que todos los sujetos expuestos a un comercial humorístico lo percibían igualmente cómico (Duncan, Nelson y Frontczak, 1983). Por esta razón, en estudios recientes acerca del humor y sus efectos se ha tenido en cuenta la per- 
cepción del humor por parte de los sujetos experimentales.

En estudios realizados por Pikett (1983), Alden (1993), Cho (1995) Aitsech, Clines y Kellaris (2003) se encontraron diferencias significativas entre los resultados obtenidos a partir del humor manipulado por los investigadores y los del humor percibido por los sujetos. Igualmente, Duncan, Nelson y Frontczak (1983) encontraron que los individuos difieren en la percepción del grado de humor presente en los comerciales. Al incluir el humor percibido como variable en dicho estudio, los autores demostraron que la comprensión del mensaje aumenta siempre y cuando el comercial que se observa sea percibido como humorístico.

En una investigación realizada por Smith (1993), se encontró que los sujetos que percibían los comerciales como humorísticos los calificaban más positivamente que aquellos a quienes no les parecían cómicos. Adicionalmente, se ha encontrado que el humor percibido se relaciona significativamente con la actitud positiva hacia la marca (Smith, 1993 y Lee, 1996) hacia el anuncio (Alden, Mukherjee y Hoyer, 2001) y la intención de compra (Smith, 1993).

\section{Humor y género}

La apreciación del humor también se ve afectada por factores de género. Es posible que los hombres no perciban un anuncio con el mismo grado de humor que las mujeres, pues sus preferencias en materia de humor difieren generalmente por razones culturales. Se ha demostrado que la respuesta al humor sexual es diferente cuando quien hace la broma (emisor) es una mujer (Gallivan, 1991).

Whipple y Courtney (1981) realizaron un estudio con hombres y mujeres practicantes de publicidad y mercadeo, encontrando diferencias significativas en sus reacciones hacia el humor en publicidad. Los hombres valoraron más positivamente que las mujeres comerciales con humor hostil. Los hallazgos demuestran que los hombres disfrutan con el humor hostil, siempre y cuando la bro- ma no sea contra los de su mismo sexo. La reacción de las mujeres frente al mismo tipo de humor resultó ser diferente a la de los hombres; no encuentran el humor hostil tan cómico como lo percibe el sexo opuesto. Este hallazgo insinúa el riesgo de la implementación del criterio propio al crear campañas humorísticas desconociendo estas diferencias de género; un tipo de humor mal utilizado puede estropear hasta la campaña más creativa.

Cantor (1976) demostró que el género de la persona que será blanco del ridículo es un determinante importante de la respuesta de humor. Los resultados de su investigación señalan que resulta más cómico ver a una mujer siendo ridiculizada que a un hombre en las mismas circunstancias. Ante esto último, el autor expone tres posibles explicaciones:

- El proceso de socialización en nuestra cultura promueve la inferioridad femenina, lo cual hace que hasta la percepción de las mujeres sobre ellas mismas se vea afectada.

- Existen expectativas culturales acerca del comportamiento apropiado de hombres y mujeres, las cuales afectan la percepción de las fuentes.

- El humor que agrede a las mujeres es más típico que el humor hostil hacia los hombres.

Por su parte, Terry y Ertel (1974) encontraron que los hombres rudos y dependientes de su grupo preferían más el humor sexual que las mujeres, especialmente las que tenían una alta inteligencia general. Por otra parte las mujeres evaluaban positivamente en mayor medida que los hombres el humor incongruente, principalmente aquéllas con baja inteligencia general. Esta relación entre preferencia de humor y género es congruente con las expectativas culturales que prohíben la opresión de hostilidad por cualquiera de los sexos y dan más libertad a los hombres en cuanto a disfrutar de estímulos relacionados con el ámbito sexual de manera pública. 


\section{Tipos de humor en publicidad}

Teniendo en cuenta que la investigación en los efectos del humor es muy reciente, los interesados en el tema han profundizado casi siempre en los mismos factores, dejando de lado algunos aspectos igualmente relevantes para la investigación en este campo tan amplio. Los tipos de humor manejados en publicidad hacen parte de los temas que vale la pena investigar, pues el hecho de que se llegue a categorizar un aspecto tan general, puede llevar a hallazgos más específicos de gran utilidad en el diseño y planeación de nuevas campañas.

En pocas investigaciones se han hecho distinciones entre tipos de humor, y algunos hallazgos basados en una sola clase de humor son generalizados a todos los tipos, lo cual claramente muestra visiones erróneas sobre la exploración del fenómeno. Es necesario tener en cuenta que, si bien existen varios tipos de humor, no significa que todos tengan el mismo efecto en la comunicación.

Un buen manejo de las técnicas de humor en la publicidad contemporánea podría llegar a hacer la diferencia entre el éxito y el fracaso de una campaña publicitaria, ya que está demostrado que ciertos tipos de humor funcionan mejor para productos específicos, así como para un medio y un público en particular (Speck, 1991; Weinberger, Campbell y Parsons, 1995; Catanescu y Tom, 2001). Aunque pocos han sido los estudios sobre la clasificación del humor, los hallazgos arrojados por las investigaciones son prometedores.

Los investigadores en el tema inician por clasificar el humor en categorías de análisis de tal manera que se puedan utilizar como variables para posteriores investigaciones en cambio de actitudes. Éste es el caso de Speck (1991), quien desarrolla una taxonomía del mensaje humorístico en la publicidad televisiva norteamericana, mediante el cruce de tipos de humor y tipos de mensaje. Define tres procesos humorísticos (Arousal-safety, incongruencia-resolución y humor despreciativo), los cuales al combinarse de diferentes maneras arrojan cinco tipos de humor característico: comic wit, humor sentimental, sátira, comedia sentimental y full comedy.

Este autor encuentra que existen diferencias significativas en los efectos de los comerciales humorísticos sobre las actitudes del consumidor dependiendo del tipo de humor que se maneje. Otro de sus hallazgos en este campo evidencia la diferencia en las preferencias en materia de humor de las culturas; encuentra que no hay un tipo de humor que tenga un impacto positivo o negativo universalmente.

Kelly y Solomon (1975) proponen que el humor puede ser categorizado al menos en dos dimensiones diferentes: contenido y/o técnica. Dentro de las tipologías de técnica, los autores citan siete clases de humor utilizados en la publicidad contemporánea norteamericana: juego de palabras (pun), bromas, subestimación, sátira, ironía, algo absurdo o ridículo, intento de humor.

Catanescu y Tom (2001) clasifican el humor de comerciales impresos y televisivos en siete categorías: comparación, personificación, exageración, juego de palabras (pun), sarcasmo, bobada (sillines) y sorpresa. Utilizando este sistema de calificación, encontraron que ciertos tipos de humor se implementan más a menudo que otros en algunos medios de comunicación.

Por su parte, Buijzen (2004) desarrolló una tipología de la publicidad humorística presente en los medios audiovisuales, encontrando siete categorías de humor: slip stick, clownish humor, sorpresa, ironía, sátira, malentendido (misunderstanding), parodia. Sus hallazgos mostraron marcadas diferencias entre las técnicas y categorías de humor dependiendo del género y del grupo de edad objetivo.

Las preferencias humorísticas se desarrollan a lo largo del tiempo, a partir de las experiencias vividas y del medio en donde se desenvuelve el sujeto. Como resultado de las diferentes preferencias humorísticas y la atención selectiva de las personas, el mismo comercial puede ser visto por varios sujetos como dos diferentes tipos de humor (Speck, 1991). 
Teniendo en cuenta que el humor está presente en todas la culturas, que es un constructo multidimensional, subjetivo y que algunos tipos de humor se utilizan mucho en ciertos países mientras que en otros poco o nunca aparecen (Speck, 1991), los resultados obtenidos en cuanto a las preferencias humorísticas de los consumidores y los efectos de ciertos tipos de humor en sus actitudes no pueden ser generalizados a nuestra cultura en particular.

La mayor parte de las investigaciones realizadas en torno al humor publicitario se ha desarrollado en un contexto norteamericano, lo cual impide realizar cualquier generalización a nuestro medio, teniendo en cuenta que el manejo de esta técnica y las preferencias humorísticas de las personas difieren de acuerdo con la cultura en la cual estén inmersos.

Por otro lado, los estudios realizados hasta ahora en este campo arrojan resultados que se basan en la publicidad humorística televisiva. Como se reportó anteriormente, el manejo de la publicidad en los medios masivos de comunicación difiere dependiendo del medio en el cual se presente. Por esta razón, es primordial generar tipologías humorísticas que se implementen en cada medio.

\section{Tipos de humor en la publicidad impresa colombiana}

Actualmente, la publicidad colombiana hace uso frecuente del humor en sus campañas, lo cual lleva a pensar que la utilización de esta técnica está dando buenos resultados, al igual que en Norteamérica. Aunque el uso del humor publicitario cada vez es más usual, no existen investigaciones acerca de los tipos de humor en la publicidad colombiana, y esto impide que se puedan elaborar campañas más efectivas diseñadas a partir de los hallazgos científicos, como sucede en otros países.

Ante la necesidad de crear herramientas que se ajusten a las características de nuestro medio en particular, se desarrolló una tipología del mensaje humorístico basada en revisiones bibliográficas y en clasificación de anuncios impresos en revistas colombianas. Considerando que la mayoría de los estudios realizados acerca de categorías de humor se ha hecho principalmente en medios televisivos, esta nueva tipología está dirigida al medio impreso.

La selección y categorización de dichos anuncios se realizó por medio de la exposición de los mismos a un grupo que debía clasificarlos teniendo en cuenta las definiciones de las categorías humorísticas implementadas por los autores referidos anteriormente. Inicialmente se identificaron siete tipos de humor, previamente citados por Catanescu y Tom (2001): sorpresa, sátira, incongruencia, juego de palabras, exageración, comparación y personificación.

Como se querían probar las categorías establecidas anteriormente, así como identificar nuevos tipos de humor publicitario, se realizó una revisión de anuncios impresos publicados en la revista Semana, desde sus inicios en 1947 hasta el 2002. Según este estudio, los tipos de humor implementados en la publicidad impresa colombiana varían de acuerdo con la época. Esto puede explicarse por el hecho de que las sociedades cambian a través del tiempo; sus necesidades, creencias y vivencias son distintas. El humor es variable y se acomoda a la sociedad en la cual se presenta.

También se encontraron diferencias en la forma de manejar las mismas categorías humorísticas dependiendo de la época en la cual se realizaron los anuncios; esto debido a que gran parte del humor se enmarca en situaciones recientes de la vida cotidiana, y muchas veces está relacionado con la memoria colectiva.

De acuerdo con los dos estudios exploratorios descritos anteriormente, los tipos de humor identificados en la publicidad impresa colombiana son:

1. Sorpresa: incluye todos los anuncios donde el humor surja de situaciones ines- 
peradas. Se considera como sorpresa cualquier situación que resulte cómica cuando algún personaje del anuncio sea sorprendido. Un ejemplo de este tipo de humor es un anuncio de Baygon, en el que los ojos de un mosquito sorprendido y asustado son resaltados en tamaño al encontrarse con el nuevo insecticida, mientras una frase es expuesta: "Usted será el segundo en notar que Baygón cambió".

2. Juego de palabras: en este tipo de humor se usan elementos del lenguaje con el fin de crear nuevos significados que resulten cómicos para el consumidor. Un anuncio de Kellogg's ejemplifica claramente esta categoría. En éste aparece la siguiente frase acompañando una caja de cereal: "Porque la única que debe ser CUADRADA es la caja. Tú no".

3. Exageración: cualquier anuncio publicitario en el que se den propiedades magnificadas o excesivas a personas, animales o cosas, puede considerarse dentro de esta categoría. En un anuncio de comida para canes Friskies, se muestra un perro que, al ser alimentado con este producto es capaz de volar varios metros por encima de la cabeza de su amo para atrapar el frisbee que le ha lanzado.

4. Comparación: anuncios en los que se comparan dos o más elementos de manera que se produzca una situación humorística. Un ejemplo de este tipo de humor comparativo es el anuncio creado para Cornina, una marca de apósito callicida. En éste aparece en la esquina izquierda la fotografía de un cortacutículas acompañado por un letrero que dice "LA GUERRA". A la derecha aparece el producto ofrecido (Cornina), seguido por la frase "LA PAZ". En este anuncio se hace una comparación entre lo que es más efectivo y menos doloroso con lo que simboliza la guerra, que en este caso es el dolor.

5. Personificación: atribución de características humanas a animales o cosas, de forma que se vuelvan cómicos. El anuncio de tarjetas de llamada de 007 Mundo que se desarrolla en un cementerio es un buen ejemplo de la personificación. En una lápida aparece dibujado un teléfono con su respectivo número en la parte inferior, dándose a entender que éste ha fallecido. El anuncio dice: "No deje morir su teléfono. Cárguelo con Tarjeta Mundo y controle el gasto de sus llamadas".

6. Caricaturas: todos los anuncios que contengan dibujo humorístico: historietas, caricaturas y dibujo animado. Esta categoría está bien ejemplificada en un anuncio de Colgate publicado en 1952. Se presenta una historieta (secuencia de imágenes dibujadas) que muestra una situación cómica: el rechazo hacia el novio por parte de una joven debido a su mal aliento.

7. Humor hostil: se contemplan dentro de esta categoría todos los anuncios en los cuales se utiliza la ironía, la parodia, el ataque y/o ridiculización de personas, animales o cosas, por medio de gestos, frases, representaciones o situaciones. Un anuncio de la revista Control TV, es un ejemplo claro del humor hostil. En él aparece un hombre con gafas, chaleco, corbata y peinado anticuado, quien sostiene una aspiradora con cara de resignación, mientras su esposa está recostada en el sofá de la sala mirándolo burlonamente. En la parte inferior del anuncio aparece un letrero que dice: “¿quién tiene el control en su casa?, para que no le queden dudas, suscríbase a Control TV'. En este comercial, el hombre es ridiculizado por no tener el control en su hogar. 
8. Incongruencia: se presenta cuando un evento no se comporta de acuerdo con nuestras expectativas con respecto a éste. Así, las personas animales o cosas en el comercial tienen actitudes o funciones que no se encuentran dentro de lo esperado normalmente. En un anuncio del portal de Internet "El Sitio", encontramos un ejemplo de incongruencia. En éste se muestra un sastre que quiere hacer uso de un serrucho para cortarle manos y pies a su cliente con el objetivo de hacer que el traje quede a su medida.

9. Otros: en esta categoría de análisis se encuentran los anuncios que no se pueden clasificar dentro de ninguno de los tipos de humor descritos anteriormente.

\section{Nuevas categorías de humor}

A partir de la revisión bibliográfica de diferentes tipologías y la recolección de anuncios humorísticos colombianos, surgieron cuatro nuevas categorías antes no identificadas dentro de la bibliografía de humor publicitario: alusiones, frases, rimas y obras.

\section{Alusiones}

Se encuentran dentro de esta categoría todos los anuncios que hagan alusión a cuentos, leyendas, mitos o historias compartidas dentro de la cultu$\mathrm{ra}$, que al relacionarse directa o indirectamente con la marca/producto/servicio publicitado resulten graciosos. En un anuncio de Vodka Finlandia, aparece el genio de la Lámpara de Aladino saliendo de la botella del licor. Una frase en la parte superior de éste dice: "No sabemos cuáles son sus tres deseos para este año"; debajo de la imagen aparece una frase un poco más grande: "Sólo sabemos hacer el mejor vodka del mundo”.
La percepción de humor en este tipo de anuncios está regida en gran parte por el conocimiento de dichas historias gracias al marco cultural en el cual se desenvuelve el sujeto. Es claro que si el consumidor no conoce la historia a la cual el anuncio hace alusión, no le hallará nada humorístico a lo que está observando. Por esta razón, es necesario delimitar muy bien el público al que va dirigido el producto que se piensa publicitar, de modo que se identifiquen las historias, mitos, leyendas o cuentos acordes con la cultura y la edad del público meta.

\section{Frases}

Esta categoría humorística se subdivide en frases originales y frases distorsionadas.

\section{- Frases originales}

Reúne todas las frases populares, creencias o refranes que hagan alusión o se refieran al producto ofrecido de una forma que resulte cómica para el consumidor. Un anuncio de Seguros Atlas muestra la imagen de un gallo en posición de escuchar, acompañado por la frase: "Más claro no canta un gallo": asegúrese para toda la vida en un santiamén.

\section{- Frases distorsionadas}

Son frases populares, creencias o refranes que han sido distorsionados de su forma original, los cuales hacen alusión o referencia al producto ofrecido de una manera cómica. La frase distorsionada se encuentra en un anuncio de celulares Ericsson, en el que se muestra la imagen en tamaño real del nuevo celular que se está ofreciendo, acompañado por la siguiente frase: "Contrario a lo que se dice, es mejor tenerlo pequeño".

Al igual que las alusiones, este tipo de humor (original y distorsionado) está relacionado directamente con la cultura de la cual hace parte el público objetivo, y requiere de un contacto, conoci- 
miento y/o utilización previa de estas frases o refranes en la cotidianidad. Ésta es una demostración más de las diferencias culturales en materia de humor.

\section{Rimas}

Conforman esta categoría aquellos anuncios publicitarios que hacen uso de palabras, frases o textos caracterizados porque su rima resulta graciosa. Como ejemplo de esta categoría se encuentra un anuncio de Foto Japón en el cual aparece la siguiente frase: " $i Y$ cómo sigue la luciérnaga el sendero si lleva la luz en el trasero?". Otro anuncio típico promociona un callicida en el que aparece la siguiente frase: "Los callos se van con callicida el Gallo. El Gallo alivia el dolor y quita el callo" (Semana, n. 298, 1952).

\section{Obras}

En esta categoría se incluyen obras artísticas (pinturas o esculturas famosas) que, al acompañarse de un elemento gráfico adicional $y / 0$ de frases, resultan cómicas para el observador. En un anuncio del Fondo Nacional de Garantías se observa al David de Miguel Ángel en primer plano, acompañado de una frase que dice: "Para obtener crédito ya no se necesita prenda".

En la mayoría de los casos, este tipo de anuncio se interpreta correctamente y es percibido como humorístico cuando el consumidor tiene conocimiento de las obras a las que hace referencia. De aquí se infiere que este tipo de humor es mejor entendido y percibido como tal por personas de un nivel cultural específico, por lo cual las campañas que lo utilizan deberían dirigirlo hacia ese sector en particular.

\section{Nuevas direcciones}

La publicidad colombiana es reconocida mundialmente por su creatividad e innovación. Frecuentemente las agencias desarrollan campañas con componente humorístico, esperando llamar la atención del consumidor y generar una actitud positiva hacia sus productos y servicios. Hasta el momento se ha utilizado el humor empíricamente como herramienta persuasiva, encontrándose una gran receptividad entre los consumidores. Ahora, si se empieza a manejar esta técnica con base en resultados de investigaciones sobre sus efectos en las actitudes y conductas, se encontrará que la efectividad del humor como técnica será todavía mayor.

Por lo tanto es importante realizar investigaciones en nuestro contexto que permitan llegar a generalizar los hallazgos obtenidos, de tal forma que se puedan aplicar a la actual publicidad colombiana. De esta manera, las campañas publicitarias humorísticas caracterizadas por la innovación y la creatividad tendrán el beneficio adicional de resultar efectivas en cuanto a los objetivos comunicacionales planteados por el cliente.

Con respecto a la investigación sobre los efectos del humor, es importante realizar estudios que hagan diferenciaciones entre género, tipo de audiencia, tipo de producto y regiones culturales. Con resultados obtenidos en el contexto colombiano, se podrán identificar técnicas humorísticas publicitarias eficaces para públicos y productos específicos. Tomando como punto de partida la tipología del humor publicitario colombiano, es posible utilizar dicha categorización para realizar estudios más específicos en las variables que han sido exploradas en otras investigaciones sin diferenciar el tipo de humor. Esto permite que los resultados sean cada vez más confiables y específicos.

Adicionalmente, resulta interesante indagar acerca de las diferencias culturales en cuanto a las preferencias por los tipos de humor.

Con la cantidad de información comercial a la que el hombre actual está expuesto diariamente, se requiere de mayor confiabilidad en las campañas publicitarias que se desarrollan; la actual competencia entre marcas es fuerte y necesita herramientas creativas para captar a los consumidores. Esta técnica publicitaria demuestra cada vez más ser una opción acertada y eficaz, lo cual nos corrobora que EL HUMOR ES COSA SERIA. 


\section{Referencias}

Alden, D. \& Hoyer, W. (1993). An Examination of Cognitive Factors Related to Humorousness in Television Advertising. Journal of Advertising. 22.

Alden.,D. Mukherjee, A.\& Hoyer, W. (2000). Extending a Contrast Resolution Model of Humor in Television Advertising: The Role of Surprise. International Journal of Humor Research, 13(2), 193-217.

Belch, G. \& Belch, M. (1984). An Investigation of the Effects of Repetition on Cognitive and Affective Reactions to Humorous and Serious Television Commercials. Advances in Consumer Research. 11, 4-10.

Berg (2001). Does Humor in Radio Advertising Affect Recognition of Novel Product Brand Names?. Journal of General Psychology, 194205.

Bryant, J. \& Zillmann, D. (1979). Teachers' Humor in the College Classroom. Communication Education. 28 110-18.

Buijzen, M. (2004). Developing a Typology of Humor in Audiovisual Media. Media Psychology. 6, 147-167.

Cantor, J. (1976). What is Funny to Whom? Journal of Communication.164-172

Cantor, J., \& Venus, P. (1980). The effects of humor on recall of a radio advertisement. Journal of Broadcasting. 24(1).

Catanescu, C. y Tom, G. (2001). Types of Humor in Television and Magazine Advertising. Review of Business, 92-95.

Chattopadhyay, A., \& Basu, K. (1990). Humor in advertising: The moderating role of prior brand evaluation. Journal of Marketing Research, 26(4), 466-476.
Cho, H. (1995). Humor Mechanisms, Perceived Humor and Their Relationships to Various Executional in Advertising. Advances in Consumer Research. 2: 191-197.

Chung, H. \& Chao, X. (2003) Humor Effect on Memory and Attitude: Moderating Role of Product Involvement. International Journal of Advertising.

Cifuentes, C. (2002). Humor Publicitario a lo Largo del Tiempo: Revista Semana (1947-1961 Y 1982-2002). Texto no publicado. Universidad de los Andes. Bogotá, Colombia.

Cline, T., Altsech, M. \& Kellaris, J. When does humor enhance or inhibit ad responses? The moderating role of the need for humor. Journal of Advertising.

Duncan, C. (1979). Humor in advertising: A behavioral perspective. Journal of the Academy of Marketing Science, 7(4), 385306

Duncan, C., Nelson, J. \& Frontczak, N. (1983). The Effect of Humor on Advertising Comprehension. Advances in Consumer Research, 11, 432-37.

Duncan, C., \& Nelson, J. (1985). Effects of humor in a radio advertising experiment. Journal of Advertising, 14, 33-40, 64.

Furnham, A., Gunter, B., \& Walsh, D. (1998). Effects of Program Context on Memory of Humorous Television Commercials. Applied Cognitive Psychology, 12, 555-567.

Gallivan, J. (1992). Group Differences in Appreciation of Feminist Humor. Humor. 5, 369-374

Gelb, B. \& Pickett, C. (1983). Attitude-towardthe-Ad: Links to Humor and to Advertising Effectiveness. Journal of Advertising, 12 (2): $34-42$. 
Gelb, B. \& Zinkhan, G.(1986). The Effect of Humor on Advertising Comprehension. Advances in Consumer Research. 11, 432 37.

Heckler, S. \& Childers, T.(1992). The role of expectancy and relevancy in memory for verbal and visual information: What is incongruency?. Journal of Consumer Research. 18, 475-492.

Holbrook, M. \& O'Shaughnessy, J. (1984). The Role of Emotion in Advertising. Psychology and Marketing. 1, 45-64.

Kaplan, R., Pascoe, G. (1977). Humorous lectures and humorous examples: Some effects upon comprehension and retention. Journal of Educational Psychology. 69, 61-65.

Kelly, J \& Solomon, P. (1975). Humor in Television Advertising. Journal of Advertising. 4, 3135.

Lammers,B., Leibowitz,L., Seymour, G \& Hennessey, J.(1983). Humor and Cognitive Responses to Advertising Stimuli: A Trace Consolidation Approach. Journal of Business Research. 11, 173-185.

Lee, J. (1996). The impact of perceived humor on advertising effects: The moderating role of consumer's evaluation to the ad and product involvement. Japanese Journal of Social Psychology. 12, 2.

Lull, P. (1940). The Effectiveness of Humor in Persuasive Speech. Communication Monographs, 7, 26-40.

Lutz, R. (1985). Affective and Cognitive Antescedents of Attitude Toward the Ad: A Conceptual Framework. Psychologycal Processes and Advertising Effects: Theory, Research and Applications. 45-56.
Lyttle, J. (2001). The Effectiveness of Humor in Persuasion: The case of Business Ethics Training._Journal of General Psychology.

MacKenzie, S., Lutz, R. (1986). The Role of Attitude Toward the Ad as Mediator of Advertising Effectiveness: A Test of Competing Explanations. Journal of Marketing Research . 23. 28-35.

Madden, T. \& Weinberger, M. (1982). The Effects of Humor on Attention in Magazine Advertising. Journal of Advertising. 11.

Madden, T y Weinberger, M. (1984). Humor in Advertising: A practitioner View. Journal of Advertising Research. 4, 23-29.

Mitchell, A. \& Olson, J. (1981). Are Product Attribute Beliefs the Only Mediator of Advertising Effects on Brand Attitude?. Journal of Marketing Research.18, 3, 318332.

Powell, J., Andersen, L. (1985) Humour and Teaching in Higher Education." Studies in Higher Education. 10, 79-89.

Ray, M. \& Batra, R. (1983). Emotion and Persuasion in Advertising: What we do and Don't Know About Affect. Advances in Consumer Research. 10, 24-38.

Scott, C., Klein, D. \& Bryant, J. (1990). Consumer Response to Humor in Advertising: A Series of Field Studies Using Behavioral Observation. JCR, 16, 498-501.

Smith, S. (1993). Does Humor in Advertising Enhance Systematic Processing?. Advances in Consumer Research. 20.

Speck, P. (1991). The Humorous Message Taxonomy. Current Issues and Research in Advertising, 13, 1-43. 
Sternthal, B. \& Craig, C. (1973). Humor in Advertising. Journal of Marketing. 37.

Stewart, D. \& Furse, D. (1986). Effective Television Advertising. Lexington, MA: DC. Health \& co; Chicago.

Sutherland, J. \& Middleton, L. (1983). The Effect of Humor on Advertising Credibility and Recall. Proceedings of the 1983 Convention of the American Academy of Advertising. 17-21.

Sylvester, A. (2000). Make'em Laugh, Make'em Buy. Advertising Age. 71 (38). 20-25.

Terry, R. y Ertel, S. (1974). Exploration of Individual Differences in Preferences for Humor. Psychologycal Reports. 1030-1037.

Weinberger, M., \& Campbell, L. (1991). The use and impact of humor in radio advertising. Journal of Advertising Research, 31, 44-52.
Weinberger, M. y Gulas, C. (1992). The impact of Humor in Advertising: A Review. Journal of Advertising, 4, 35-55.

Weinberger, M. Spots, H. Campbell, L. y Parsons, A. (1995). The use and Effect of Humor in Different Advertising Media. Journal of Advertising Research. 44-54.

Whipple,T. y Courtney,A. (1981) How Men and Women Judge Humor: Advertising Guidelines for Action Research. Current Issues and Research in Advertising_43-54.

Zhang, Y. \& Zinkhan, G. (1991). "Humor in Television Advertising: The Effects of Repetition and Social Setting. Advances in Consumer Research. 18, 813-818.

Zhang, Y. (1996). Responses to Humorous Advertising: The moderating effect of need for cognition. Journal of Advertising 